\title{
On the connection between Stirling numbers and Bessel numbers
}

\author{
David Stenlund* \\ Faculty of Science and Engineering \\ Åbo Akademi University \\ Turku, Finland \\ david.stenlund@abo.fi
}

Submitted: Sept 3, 2020; Accepted: Oct 21, 2021; Published: Feb 25, 2022

(C) The author. Released under the CC BY license (International 4.0).

\begin{abstract}
We present new proofs for some summation identities involving Stirling numbers of both first and second kind. The two main identities show a connection between Stirling numbers and Bessel numbers. Our method is based on solving a particular recurrence relation in two different ways and comparing the coefficients in the resulting polynomial expressions. We also briefly discuss a probabilistic setting where this recurrence relation occurs.
\end{abstract}

Mathematics Subject Classifications: 11B73, 11B83, 05A19, 60J60

\section{Introduction}

Stirling numbers of the first and second kind are well-known numbers that are found in numerous combinatorial problems. A combinatorial interpretation of these numbers is that the unsigned Stirling number of the first kind $\left[\begin{array}{l}n \\ k\end{array}\right]$ counts the number of permutations of $n$ elements with $k$ disjoint cycles, while the Stirling number of the second kind $\left\{\begin{array}{l}n \\ k\end{array}\right\}$ corresponds to the number of ways to partition a set of $n$ elements into $k$ nonempty subsets. The numbers $(-1)^{n-k}\left[\begin{array}{l}n \\ k\end{array}\right]$ are called signed Stirling numbers of the first kind.

In this paper, we consider sums containing both kinds of Stirling numbers of the form

$$
\sum_{i=k}^{n}\left[\begin{array}{l}
n \\
i
\end{array}\right]\left\{\begin{array}{l}
i \\
k
\end{array}\right\} z^{i},
$$

for certain special values of $z \in \mathbb{R}$. Of particular interest are the following two results that connect such sums with either the first or the second kind of Bessel numbers, denoted $b(n, k)$ and $B(n, k)$, respectively.

\footnotetext{
*Supported by a grant from the Magnus Ehrnrooth Foundation.
} 
Theorem 1. For any $n, k \in \mathbb{Z}_{+}$,

$$
\sum_{i=k}^{n}\left[\begin{array}{l}
n \\
i
\end{array}\right]\left\{\begin{array}{l}
i \\
k
\end{array}\right\}(-2)^{n-i}=b(n, k) .
$$

Theorem 2. For any $n, k \in \mathbb{Z}_{+}$,

$$
\sum_{i=k}^{n}\left[\begin{array}{c}
n \\
i
\end{array}\right]\left\{\begin{array}{l}
i \\
k
\end{array}\right\}(-2)^{i-k}=(-1)^{n-k} B(n, k) .
$$

In Section 2, we define and discuss the Stirling and Bessel numbers. New proofs for the identities in Theorems 1 and 2 are presented in Section 3. The identities are derived by solving a particular recurrence relation in two ways and thereafter equating the coefficients in the resulting polynomial expressions. In this way, the proofs are of a rather elementary nature, although there is some algebra involved. In Section 4, the same method is used to derive two other known summation identities, as well as expressions for the sum in (1) with $z$ being a negative integer. A few other possibilities are also suggested.

An additional aim of this paper is to highlight a probabilistic setting where Theorem 1 applies. The recurrence relation that is used as basis for the proof is in fact a recursive formula for the moments of the occupation time on $[0, \infty)$ for a skew two-sided Bessel process. In Section 5, we briefly describe the occupation time and explain the interpretation of Theorem 1 in terms of skew two-sided Bessel processes and the special case of skew Brownian motion.

\section{Stirling numbers and Bessel numbers}

Unsigned Stirling numbers of the first kind $\left[\begin{array}{l}n \\ k\end{array}\right]$ and Stirling numbers of the second kind $\left\{\begin{array}{l}n \\ k\end{array}\right\}$ are defined recursively for all $n, k \in \mathbb{Z}$ through

$$
\left[\begin{array}{c}
n+1 \\
k
\end{array}\right]=n\left[\begin{array}{l}
n \\
k
\end{array}\right]+\left[\begin{array}{c}
n \\
k-1
\end{array}\right] \text { and }\left\{\begin{array}{c}
n+1 \\
k
\end{array}\right\}=k\left\{\begin{array}{l}
n \\
k
\end{array}\right\}+\left\{\begin{array}{c}
n \\
k-1
\end{array}\right\}
$$

with initial conditions

$$
\left[\begin{array}{l}
n \\
0
\end{array}\right]=\left\{\begin{array}{l}
n \\
0
\end{array}\right\}=\delta_{n, 0}, \quad\left[\begin{array}{l}
0 \\
k
\end{array}\right]=\left\{\begin{array}{l}
0 \\
k
\end{array}\right\}=\delta_{0, k},
$$

where $\delta$ is the Kronecker delta. Another way of defining the unsigned Stirling numbers of the first kind is that they are the coefficients of a rising factorial, [6, Eq. (6.11)]

$$
(x)_{n}=x(x+1) \cdots(x+n-1)=\sum_{k=0}^{n}\left[\begin{array}{l}
n \\
k
\end{array}\right] x^{k},
$$

for $n \in \mathbb{N}$ and with $(x)_{0}=1$. Similarly, Stirling numbers of the second kind are the coefficients when ordinary powers are expressed using falling factorials, [6, Eq. (6.10)]

$$
x^{n}=\sum_{k=0}^{n}\left\{\begin{array}{l}
n \\
k
\end{array}\right\} x(x-1) \cdots(x-k+1) .
$$


The notation for Stirling numbers varies. A rather common notation is $s(n, k)$ and $S(n, k)$ for Stirling numbers of the first and second kind, respectively. This notation is used, for instance, by Comtet [4] and Mansour and Schork [10]. However, here we follow the notation used by Graham, Knuth and Patashnik [6] (see also Knuth's remarks on the subject [8]), and refer to their book for basic properties of Stirling numbers, including a number of useful summation identities.

The Bessel polynomials are a sequence of polynomials that are solutions to the second order differential equation

$$
x^{2} y_{n}^{\prime \prime}(x)+(2 x+2) y_{n}^{\prime}(x)-n(n+1) y_{n}(x)=0,
$$

with the normalization $y_{n}(0)=1$. The first few Bessel polynomials are

$$
\begin{aligned}
& y_{0}(x)=1, \\
& y_{1}(x)=x+1, \\
& y_{2}(x)=3 x^{2}+3 x+1, \\
& y_{3}(x)=15 x^{3}+15 x^{2}+6 x+1, \\
& y_{4}(x)=105 x^{4}+105 x^{3}+45 x^{2}+10 x+1 .
\end{aligned}
$$

Sometimes the polynomials with coefficients in opposite order are used instead. These are consequently called reverse Bessel polynomials, and are given by

$$
\theta_{n}(x):=x^{n} y_{n}(1 / x) .
$$

Both these types of Bessel polynomials may also be defined recursively, namely

$$
\begin{aligned}
& y_{0}(x)=1, \quad y_{1}(x)=x+1, \quad y_{n}(x)=(2 n-1) x y_{n-1}(x)+y_{n-2}(x), \\
& \theta_{0}(x)=1, \quad \theta_{1}(x)=x+1, \quad \theta_{n}(x)=(2 n-1) \theta_{n-1}(x)+x^{2} \theta_{n-2}(x) \text {. }
\end{aligned}
$$

The name Bessel polynomials was first used by Krall and Frink [9] due to the connection to Bessel functions. Indeed, the Bessel polynomials can also be written

$$
y_{n}(x)=\sqrt{\frac{2}{\pi x}} e^{1 / x} K_{n+\frac{1}{2}}(1 / x),
$$

where $K_{\nu}(x)$ is a modified Bessel function of the second kind. We refer to Grosswald [7] for more properties of Bessel polynomials.

The (signed) Bessel number of the first kind $b(n, k)$ is defined as the coefficient before $x^{n-k}$ in the polynomial $y_{n-1}(-x)$. Explicitly, this number is given for $1 \leqslant k \leqslant n$ by

$$
b(n, k)=(-1)^{n-k} \frac{(2 n-k-1) !}{2^{n-k}(k-1) !(n-k) !} .
$$

The Bessel number of the second kind $B(n, k)$ is given for $\left\lceil\frac{n}{2}\right\rceil \leqslant k \leqslant n$ by

$$
B(n, k)=\frac{n !}{2^{n-k}(2 k-n) !(n-k) !} .
$$


These numbers are related through $(-1)^{n-k} B(n, k)=b(k+1,2 k-n+1)$, and they form a dual pair in the sense that [14, Eq. (11)-(12)]

$$
\sum_{k=m}^{n} B(n, k) b(k, m)=\sum_{k=m}^{n} b(n, k) B(k, m)=\delta_{m, n}
$$

for any $m, n \in \mathbb{Z}_{+}$. A similar duality will be shown also for Stirling numbers, see (18).

It has earlier been shown that the sum in (1) can be expressed using degenerate Stirling numbers [14, Eq. (24)-(25)] or generalized Stirling numbers [10, Prop. 8.129] (see also (23) in Section 5 below). These are two examples of extended versions of the Stirling numbers, and in [10] are listed several more variations.

Theorems 1 and 2 provide a link between Stirling numbers (of both kinds) and Bessel numbers of either the first or the second kind. The identities are proved by Yang and Qiao in [14] using exponential Riordan arrays. Both identities are also special cases of the formula [11, Prop. 16]

$$
\mathfrak{S}_{\frac{s}{\nu} ; \nu}(n, k)=\sum_{i=k}^{n} \mathfrak{S}_{\frac{s}{\nu-\sigma} ; \nu-\sigma}(n, i) \mathfrak{S}_{\frac{s+\sigma-\nu}{\sigma} ; \sigma}(i, k),
$$

valid for $s \in \mathbb{R}, \nu \neq 0, \sigma>0$, where $\mathfrak{S}_{s, h}(n, k)$ are generalized Stirling numbers defined recursively for $s \in \mathbb{R}$ and $h \in \mathbb{C} \backslash\{0\}$ through

$$
\begin{gathered}
\mathfrak{S}_{s ; h}(n+1, k)=\mathfrak{S}_{s ; h}(n, k-1)+h(k+s(n-k)) \mathfrak{S}_{s ; h}(n, k), \\
\mathfrak{S}_{s ; h}(n, 0)=\delta_{n, 0}, \quad \mathfrak{S}_{s ; h}(0, k)=\delta_{0, k} .
\end{gathered}
$$

Comparing the more general recursion above to those for Stirling numbers, we note that $\mathfrak{S}_{1 ; 1}(n, k)=\left[\begin{array}{l}n \\ k\end{array}\right]$ and $\mathfrak{S}_{0 ; 1}(n, k)=\left\{\begin{array}{l}n \\ k\end{array}\right\}$. Bessel numbers are also special cases of generalized Stirling numbers, namely $\mathfrak{S}_{2 ;-1}(n, k)=b(n, k)$ and $\mathfrak{S}_{-1 ; 1}(n, k)=B(n, k)$. Using this, and the fact that

$$
\mathfrak{S}_{s ; a h}(n, k)=a^{n-k} \mathfrak{S}_{s ; h}(n, k)
$$

we see that insertion of $(s, \nu, \sigma)=(-2,-1,1)$ into (9) gives precisely (2), while insertion of $(s, \nu, \sigma)=\left(-\frac{1}{2}, \frac{1}{2}, 1\right)$ gives $(3)$.

Our proofs for the identities (2) and (3) were found independently and initially unknowingly of these previously known results. For more details on generalized Stirling numbers, as well as further references, see Mansour and Schork [10]. In the following, we will for the most part not consider the generalized versions but rather use ordinary Stirling numbers.

\section{Proof of the main identities}

Consider the following polynomial recurrence relation for $n \geqslant 1$ and $x, z \in \mathbb{R}$ :

$$
P_{1}(x, z)=x, \quad P_{n+1}(x, z)=x\left(\begin{array}{c}
n+z \\
n
\end{array}\right)-x \sum_{k=1}^{n}\left(\begin{array}{c}
n-k+z \\
n-k+1
\end{array}\right) P_{k}(x, z) .
$$


The reason behind studying this particular recurrence relation is explained in Section 5 . Salminen and Stenlund [12] prove the following solution.

Lemma 3. For any $n \geqslant 1$,

$$
P_{n}(x, z)=\sum_{i=1}^{n} \sum_{k=1}^{i} \frac{(-1)^{k-1}(k-1) !}{(n-1) !}\left[\begin{array}{c}
n \\
i
\end{array}\right]\left\{\begin{array}{l}
i \\
k
\end{array}\right\} x^{k} z^{i-1}
$$

is a solution to (10).

Proof (sketch). For a more detailed proof we refer to Salminen and Stenlund [12, Thm. 4]. Note that neither of the assumptions $x \in(0,1)$ and $z \in(-1,0)$ that are implicit therein (due to the setting of skew two-sided Bessel processes) is necessary for this proof, and thus the result holds without these restrictions on $x$ and $z$. The case $z=0$ is trivial, since $P_{n}(x, 0)=x$ for all $n$, and thus we can hereafter assume that $z \neq 0$. Recall that the result is proved by induction. First, the binomial coefficient inside the sum in (10) is rewritten as

$$
\left(\begin{array}{c}
n-m+z \\
n-m+1
\end{array}\right)=\frac{1}{(n-m+1) !} \sum_{i=m}^{n}\left[\begin{array}{c}
n-m+1 \\
n-i+1
\end{array}\right] z^{n-i+1},
$$

based on (4). Thereafter, the induction assumption is applied, by inserting the expression in (11) for $P_{m}(x, z)$ into (10), which yields a quadruple sum. After changing the summation order, this expression is simplified in a suitable way until the desired form as in (11) is obtained for $P_{n+1}(x, z)$, and the result follows by induction. Key identities for the simplification steps are

$$
\left[\begin{array}{c}
n+1 \\
i
\end{array}\right]\left(\begin{array}{c}
i-1 \\
j-2
\end{array}\right)=\sum_{m=j-1}^{n-i+j}\left[\begin{array}{c}
m \\
j-1
\end{array}\right]\left[\begin{array}{c}
n-m+1 \\
i-j+1
\end{array}\right]\left(\begin{array}{c}
n \\
m-1
\end{array}\right)
$$

and

$$
\sum_{j=i}^{k}\left\{\begin{array}{l}
j-1 \\
i-1
\end{array}\right\}\left(\begin{array}{l}
k-1 \\
j-2
\end{array}\right)=(i-1)\left\{\begin{array}{l}
k \\
i
\end{array}\right\}
$$

which are modified versions of equations (6.29) and (6.15) in [6], respectively.

The solution to the recurrence relation in (10) is a polynomial in $x$ and $z$ with Stirling numbers of both first and second kind in the coefficients. For certain fixed values of $z$, the relation (10) can be solved in a different way than in the proof of Lemma 3 to obtain another polynomial expression for the solution. Equating the coefficients in these alternative solutions then leads to a summation identity for Stirling numbers of both kinds. In particular, Theorems 1 and 2 can be proved this way.

Proof of Theorem 1. For this proof we consider the case when $z=-1 / 2$ (which is also of particular interest due to the probabilistic interpretation presented in Section 5). Inserting this value into (10) and recalling that

$$
\left(\begin{array}{c}
n-\frac{1}{2} \\
n
\end{array}\right)=2^{-2 n}\left(\begin{array}{c}
2 n \\
n
\end{array}\right)
$$


we get the recurrence relation

$$
\begin{aligned}
P_{n+1}\left(x,-\frac{1}{2}\right) & =\frac{x}{2^{2 n}}\left(\begin{array}{c}
2 n \\
n
\end{array}\right)+\sum_{i=1}^{n} \frac{x}{2^{2 n-2 i+1}(n-i+1)}\left(\begin{array}{c}
2 n-2 i \\
n-i
\end{array}\right) P_{i}\left(x,-\frac{1}{2}\right) \\
& =\frac{x}{2^{2 n}}\left(\begin{array}{c}
2 n \\
n
\end{array}\right)+\sum_{i=0}^{n-1} \frac{x}{2^{2 i+1}(i+1)}\left(\begin{array}{c}
2 i \\
i
\end{array}\right) P_{n-i}\left(x,-\frac{1}{2}\right) .
\end{aligned}
$$

As this is a special case of (10), a solution is given by (11) with $z=-1 / 2$. However, using another proof by induction, we show that for $n \geqslant 1$, the polynomial

$$
P_{n}\left(x,-\frac{1}{2}\right)=\sum_{k=0}^{n-1}\left(\begin{array}{c}
n+k-1 \\
k
\end{array}\right) \frac{x^{n-k}}{2^{n+k-1}}
$$

solves (12). Clearly, the identity holds for $n=1$. Assume now that (13) holds for all $n \in\{1,2, \ldots, N\}$. It then follows from (12) that

$$
\begin{aligned}
P_{N+1}\left(x,-\frac{1}{2}\right) & =\frac{x}{2^{2 N}}\left(\begin{array}{c}
2 N \\
N
\end{array}\right)+\sum_{i=0}^{N-1} \frac{x}{2^{2 i+1}(i+1)}\left(\begin{array}{c}
2 i \\
i
\end{array}\right) \sum_{k=0}^{N-i-1}\left(\begin{array}{c}
N-i+k-1 \\
k
\end{array}\right) \frac{x^{N-i-k}}{2^{N-i+k-1}} \\
& =\frac{x}{2^{2 N}}\left(\begin{array}{c}
2 N \\
N
\end{array}\right)+\sum_{i=0}^{N-1} \sum_{k=i}^{N-1} \frac{x^{N+1-k}}{2^{N+k}(i+1)}\left(\begin{array}{c}
2 i \\
i
\end{array}\right)\left(\begin{array}{c}
N-2 i+k-1 \\
k-i
\end{array}\right) \\
& =\frac{x}{2^{2 N}}\left(\begin{array}{c}
2 N \\
N
\end{array}\right)+\sum_{k=0}^{N-1} \frac{x^{N+1-k}}{2^{N+k}} \sum_{i=0}^{k} \frac{1}{2 i+1}\left(\begin{array}{c}
2 i+1 \\
i
\end{array}\right)\left(\begin{array}{c}
N+k-1-2 i \\
k-i
\end{array}\right),
\end{aligned}
$$

and the inner sum can be evaluated using the Hagen-Rothe identity

$$
\sum_{k=0}^{n} \frac{a}{a+b k}\left(\begin{array}{c}
a+b k \\
k
\end{array}\right)\left(\begin{array}{c}
c-b k \\
n-k
\end{array}\right)=\left(\begin{array}{c}
a+c \\
n
\end{array}\right),
$$

which is a special case of Equation (3.146) in [5] (see also [3]). This yields

$$
\begin{aligned}
P_{N+1}\left(x,-\frac{1}{2}\right) & =\frac{x}{2^{2 N}}\left(\begin{array}{c}
2 N \\
N
\end{array}\right)+\sum_{k=0}^{N-1}\left(\begin{array}{c}
N+k \\
k
\end{array}\right) \frac{x^{N+1-k}}{2^{N+k}} \\
& =\sum_{k=0}^{N}\left(\begin{array}{c}
N+k \\
k
\end{array}\right) \frac{x^{N+1-k}}{2^{N+k}},
\end{aligned}
$$

and thus, by induction, (13) holds for all $n \geqslant 1$. On the other hand, inserting $z=-1 / 2$ into (11) gives that

$$
P_{n}\left(x,-\frac{1}{2}\right)=\sum_{k=1}^{n} x^{k} \frac{(-1)^{k-1}(k-1) !}{(n-1) !} \sum_{i=k}^{n}\left[\begin{array}{l}
n \\
i
\end{array}\right]\left\{\begin{array}{l}
i \\
k
\end{array}\right\}(-2)^{1-i} .
$$


Equating the coefficients before $x^{k}$ in (13) and (14) we obtain

$$
\frac{(-1)^{k-1}(k-1) !}{(n-1) !} \sum_{i=k}^{n}\left[\begin{array}{l}
n \\
i
\end{array}\right]\left\{\begin{array}{l}
i \\
k
\end{array}\right\}(-2)^{1-i}=\frac{1}{2^{2 n-k-1}}\left(\begin{array}{c}
2 n-k-1 \\
n-k
\end{array}\right), \quad 1 \leqslant k \leqslant n,
$$

which is equivalent to (2), and the proof is complete.

Proof of Theorem 2. In this case we let $z=-2$. Inserting $n=1$ into (10) we see that

$$
P_{2}(x,-2)=-x-x\left(-2 P_{1}(x,-2)\right)=2 x^{2}-x,
$$

which becomes our second initial condition, together with $P_{1}(x,-2)=x$. For any $n \geqslant 3$ the first term disappears altogether, and only two terms are left in the sum. The recurrence relation thus becomes

$$
P_{n+1}(x,-2)=x\left(2 P_{n}(x,-2)-P_{n-1}(x,-2)\right) .
$$

This recurrence relation can be solved in a standard way. The characteristic equation

$$
1-2 u+\frac{u^{2}}{x}=0
$$

has the roots $u=x \pm \sqrt{x(x-1)}$. Note that we are only using the variable $x$ for comparing the polynomial coefficients, so we can assume here that $x>1$. A solution to the recurrence relation is then

$$
P_{n}(x,-2)=c_{1}(x+\sqrt{x(x-1)})^{n}+c_{2}(x-\sqrt{x(x-1)})^{n},
$$

and from the initial conditions it is seen that $c_{1}=c_{2}=\frac{1}{2}$. Expanding both binomial expressions as sums and combining them we obtain

$$
\begin{aligned}
P_{n}(x,-2) & =\sum_{i=0}^{n}\left(\begin{array}{c}
n \\
i
\end{array}\right) x^{n-i}(x(x-1))^{\frac{i}{2}}\left(\frac{1+(-1)^{i}}{2}\right) \\
& =\sum_{m=0}^{\left\lfloor\frac{n}{2}\right\rfloor}\left(\begin{array}{c}
n \\
2 m
\end{array}\right) x^{n-m}(x-1)^{m} \\
& =\sum_{m=0}^{\left\lfloor\frac{n}{2}\right\rfloor} \sum_{k=0}^{m}(-1)^{k}\left(\begin{array}{c}
n \\
2 m
\end{array}\right)\left(\begin{array}{c}
m \\
k
\end{array}\right) x^{n-k} \\
& =\sum_{k=0}^{\left\lfloor\frac{n}{2}\right\rfloor}(-1)^{k} 2^{n-2 k-1}\left(\begin{array}{c}
n-k \\
k
\end{array}\right) \frac{n}{n-k} x^{n-k} \\
& =\frac{n}{2} \sum_{k=\left\lceil\frac{n}{2}\right\rceil}^{n}(-1)^{n-k} \frac{(k-1) ! 2^{2 k-n}}{(n-k) !(2 k-n) !} x^{k},
\end{aligned}
$$


where in the second to last step the identity [5, Eq. (3.120)]

$$
\sum_{m=k}^{\left\lfloor\frac{n}{2}\right\rfloor}\left(\begin{array}{c}
n \\
2 m
\end{array}\right)\left(\begin{array}{c}
m \\
k
\end{array}\right)=2^{n-2 k-1}\left(\begin{array}{c}
n-k \\
k
\end{array}\right) \frac{n}{n-k}
$$

is used. An alternative way of obtaining (16) is to recognize the similarity between the recurrence relation in (15) and that of the Chebyshev polynomials of the first kind $T_{n}(x)$. These polynomials are defined recursively through

$$
T_{0}(x)=1, \quad T_{1}(x)=x, \quad T_{n+1}(x)=2 x T_{n}(x)-T_{n-1}(x) .
$$

In fact, we see that $P_{n}(x,-2)=(\sqrt{x})^{n} T_{n}(\sqrt{x})$ satisfies (15), and from an explicit expression for Chebyshev polynomials $[1,(22.3 .6)]$ we then get (16). What remains of the proof is just to compare the coefficients before $x^{k}$ in (11) when $z=-2$ with those in (16). This gives

$$
\frac{(-1)^{k-1}(k-1) !}{(n-1) !} \sum_{i=k}^{n}\left[\begin{array}{l}
n \\
i
\end{array}\right]\left\{\begin{array}{l}
i \\
k
\end{array}\right\}(-2)^{i-1}= \begin{cases}(-1)^{n-k} \frac{n(k-1) ! 2^{2 k-n-1}}{(n-k) !(2 k-n) !}, & \left\lceil\frac{n}{2}\right\rceil \leqslant k \leqslant n \\
0, & \text { otherwise }\end{cases}
$$

which is equivalent to $(3)$.

\section{Extension to other identities}

As we have seen in the previous section, the choice of $z=-1 / 2$ or $z=-2$ in (10) results in an identity connecting Stirling numbers with Bessel numbers of the first or second kind, respectively. Here we consider some other choices of $z$ that give rise to similar summation identities.

When $z$ is an integer, the binomial coefficients in (10) are also integers, and thereby the polynomials $P_{n}(x, z)$ have integer coefficients. A simple example is $z=1$, for which the recurrence relation becomes

$$
P_{1}(x, 1)=x, \quad P_{n+1}(x, 1)=(n+1) x-x \sum_{k=1}^{n} P_{k}(x, 1) .
$$

In this case it is easy to prove by induction that, for all $n \geqslant 1$,

$$
P_{n}(x, 1)=1-(1-x)^{n}=\sum_{k=1}^{n}(-1)^{k+1}\left(\begin{array}{l}
n \\
k
\end{array}\right) x^{k} .
$$

When compared to (11), this yields the known identity

$$
\sum_{i=k}^{n}\left[\begin{array}{l}
n \\
i
\end{array}\right]\left\{\begin{array}{l}
i \\
k
\end{array}\right\}=\frac{(n-1) !}{(k-1) !}\left(\begin{array}{l}
n \\
k
\end{array}\right)=: L(n, k),
$$


where $L(n, k)$ are so called Lah numbers [4, p. 156]. The method presented here could potentially be used to find identities for other positive integer values of $z$ as well, although the recurrence relations for $z \geqslant 2$ are not quite as simple as when $z=1$. Solving the recurrence relation for half-integer values also seems a viable possibility, as the case $z=-1 / 2$ is already done in the proof of Theorem 1 . This is worth investigating further.

When $z$ is a negative integer $-m$, there are at most $m$ terms on the right hand side of (10). After the first $m$ steps of the recursion, we have a (homogeneous) linear recurrence relation of order $m$. An example of this has already been illustrated in the proof of Theorem 2 with $z=-2$, and we proceed to derive a result for other negative integer values of $z$ using the same method.

The case $z=-1$ is trivial, since

$$
P_{1}(x,-1)=x, \quad P_{n+1}(x,-1)=x P_{n}(x,-1),
$$

and thus $P_{n}(x,-1)=x^{n}$ for all $n \geqslant 1$. Comparing this to (11) with $z=-1$ leads to the well-known inversion formula for Stirling numbers,

$$
\sum_{i=k}^{n}\left[\begin{array}{l}
n \\
i
\end{array}\right]\left\{\begin{array}{l}
i \\
k
\end{array}\right\}(-1)^{n-i}=\delta_{n, k},
$$

which is given, for instance, in [6, p. 264]. Compare this to the corresponding identity (8) for Bessel numbers.

Let $z=-m$ be any negative integer $\left(m \in \mathbb{Z}_{+}\right)$. The binomial coefficient in the first term of (10) becomes

$$
\left(\begin{array}{c}
n-m \\
n
\end{array}\right)= \begin{cases}(-1)^{n}\left(\begin{array}{c}
m-1 \\
n
\end{array}\right), & 0 \leqslant n<m \\
0, & 0<m \leqslant n\end{cases}
$$

using the well-known formula for negating the upper index of a binomial coefficient $[6$, Eq. (5.14)]. Similarly, the binomial coefficient inside the sum is zero whenever $k \leqslant n-m$. The recurrence relation becomes

$$
P_{n}(x,-m)= \begin{cases}(-1)^{n+1} x\left(\left(\begin{array}{c}
m-1 \\
n-1
\end{array}\right)+\sum_{k=1}^{n-1}(-1)^{k}\left(\begin{array}{c}
m \\
n-k
\end{array}\right) P_{k}(x,-m)\right), & 1 \leqslant n \leqslant m, \\
(-1)^{n+1} x \sum_{k=n-m}^{n-1}(-1)^{k}\left(\begin{array}{c}
m \\
n-k
\end{array}\right) P_{k}(x,-m), & n>m .\end{cases}
$$

This can be solved using standard methods. The characteristic equation is

$$
u^{m}\left(\frac{1}{x}-1\right)+(u-1)^{m}=0
$$

and the roots are given by

$$
u_{k}=\frac{1}{1-\omega^{k}(1-1 / x)^{\frac{1}{m}}}=x \sum_{j=0}^{m-1} \omega^{k j}(1-1 / x)^{\frac{j}{m}}, \quad k=0, \ldots, m-1,
$$


where $\omega=e^{i 2 \pi / m}$ is a primitive $m$ th root of unity. The general solution to the recurrence becomes

$$
P_{n}(x,-m)=\frac{1}{m} \sum_{k=0}^{m-1} u_{k}^{n}=\frac{x^{n}}{m} \sum_{k=0}^{m-1}\left(\sum_{j=0}^{m-1} \omega^{k j}(1-1 / x)^{\frac{j}{m}}\right)^{n} .
$$

Let $c_{m, n, j}$ be the coefficient before $x^{j}$ in the expansion of $\left(1+x+x^{2}+\cdots+x^{m-1}\right)^{n}$. This number is given by

$$
\begin{aligned}
c_{m, n, j} & =\sum_{\substack{k_{1}+k_{2}+\cdots+k_{m}=n \\
k_{1}+2 k_{2}+\cdots+m k_{m}=n+j}} \frac{n !}{k_{1} ! k_{2} ! \cdots k_{m} !} \\
& =\sum_{k_{1}=0}^{n} \sum_{k_{2}=0}^{k_{1}} \cdots \sum_{k_{m-2}=0}^{k_{m-3}}\left(\begin{array}{l}
n \\
k_{1}
\end{array}\right)\left(\begin{array}{l}
k_{1} \\
k_{2}
\end{array}\right) \cdots\left(\begin{array}{c}
k_{m-3} \\
k_{m-2}
\end{array}\right)\left(\begin{array}{c}
k_{m-2} \\
j-\left(k_{1}+\cdots+k_{m-2}\right)
\end{array}\right) .
\end{aligned}
$$

We now write

$$
\begin{aligned}
P_{n}(x,-m) & =\frac{x^{n}}{m} \sum_{k=0}^{m-1} \sum_{j=0}^{(m-1) n} c_{m, n, j}\left(\omega^{k}(1-1 / x)^{\frac{1}{m}}\right)^{j} \\
& =\frac{x^{n}}{m} \sum_{j=0}^{(m-1) n} c_{m, n, j}(1-1 / x)^{\frac{j}{m}} \sum_{k=0}^{m-1} \omega^{k j},
\end{aligned}
$$

and, since $\omega$ is an $m$ th root of unity,

$$
\sum_{k=0}^{m-1} \omega^{k j}= \begin{cases}m, & j=m N \text { for some } N \in \mathbb{N} \\ 0, & \text { otherwise }\end{cases}
$$

so we get that

$$
\begin{aligned}
P_{n}(x,-m) & =x^{n} \sum_{N=0}^{n} c_{m, n, m N}(1-1 / x)^{N} \\
& =\sum_{N=0}^{n} \sum_{k=n-N}^{n} c_{m, n, m N}(-1)^{n-k}\left(\begin{array}{c}
N \\
n-k
\end{array}\right) x^{k} .
\end{aligned}
$$

Note that the upper summation limit for $N$ should actually be $\left\lfloor n-\frac{n}{m}\right\rfloor$, but $c_{m, n, m N}=0$ for any $N>\left\lfloor n-\frac{n}{m}\right\rfloor$, so we may choose $n$ as the upper summation limit for simplicity. When comparing the coefficients before $x^{k}$ in (20) with those in (11) when $z=-m$, we obtain the following result.

Theorem 4. For any $n, k, m \in \mathbb{Z}_{+}$,

$$
\sum_{i=k}^{n}\left[\begin{array}{c}
n \\
i
\end{array}\right]\left\{\begin{array}{l}
i \\
k
\end{array}\right\}(-m)^{i}= \begin{cases}\frac{(-1)^{n} m(n-1) !}{(k-1) !} \sum_{N=n-k}^{n} c_{m, n, m N}\left(\begin{array}{c}
N \\
n-k
\end{array}\right), & \left\lceil\frac{n}{m}\right\rceil \leqslant k \leqslant n \\
0, & \text { otherwise }\end{cases}
$$

where $c_{m, n, m N}$ is given by (19). 
We have already seen expressions for the cases $m=1$ and $m=2$. Similar identities can be obtained for other values of $m$ using the result above, and to illustrate this we give one more example. Inserting $m=3$ into (19) gives

$$
c_{3, n, 3 N}=\sum_{j=0}^{n}\left(\begin{array}{l}
n \\
j
\end{array}\right)\left(\begin{array}{c}
j \\
3 N-j
\end{array}\right)
$$

and from (21) we get the following formula.

Corollary 5. For any $n, k \in \mathbb{Z}_{+}$with $\left\lceil\frac{n}{3}\right\rceil \leqslant k \leqslant n$,

$$
\sum_{i=k}^{n}\left[\begin{array}{l}
n \\
i
\end{array}\right]\left\{\begin{array}{l}
i \\
k
\end{array}\right\}(-3)^{i}=\frac{(-1)^{n} 3(n-1) !}{(k-1) !} \sum_{j=0}^{n} \sum_{N=n-k}^{n}\left(\begin{array}{l}
n \\
j
\end{array}\right)\left(\begin{array}{c}
j \\
3 N-j
\end{array}\right)\left(\begin{array}{c}
N \\
n-k
\end{array}\right) .
$$

Remark 6. An alternative approach is to use generating functions to derive the results above. From the exponential generating functions for Stirling numbers of the first and second kind we can obtain the exponential generating function for the sum in (1) as

$$
\sum_{n=0}^{\infty} \frac{w^{n}}{n !} \sum_{i=k}^{n}\left[\begin{array}{l}
n \\
i
\end{array}\right]\left\{\begin{array}{l}
i \\
k
\end{array}\right\} z^{i}=\frac{\left((1-w)^{-z}-1\right)^{k}}{k}
$$

Inserting $z=-1 / 2$ or $z=-2$, and after a slight modification, the result is recognized as the exponential generating function for Bessel numbers of the first or second kind, respectively. Equations (17) and (18) can also be derived similarly. Furthermore, letting $z=-3$ leads to the formula

$$
\sum_{i=k}^{n}\left[\begin{array}{c}
n \\
i
\end{array}\right]\left\{\begin{array}{l}
i \\
k
\end{array}\right\}(-3)^{i}=(-1)^{k} \frac{n !}{k !} \sum_{m=\max \{0, n-2 k\}}^{\min \{k, n-k\}}\left(\begin{array}{c}
k \\
m
\end{array}\right)\left(\begin{array}{c}
k \\
n-k-m
\end{array}\right)\left(-w_{1}\right)^{k-m}\left(-w_{2}\right)^{n-k}
$$

where

$$
w_{1}=\frac{3+\mathrm{i} \sqrt{3}}{2}, \quad w_{2}=\frac{3-\mathrm{i} \sqrt{3}}{2} .
$$

The use of generating functions is often a versatile method, and complements the earlier discussion in this paper. Nevertheless, the deliberate choice here is to focus on the approach of solving the recurrence relation (10) to highlight the fact that the relation and its solution appear in a certain probabilistic setting, which is presented in the following section.

\section{A probabilistic interpretation}

The significance of the recursive equation (10) is that it arises in the study of the occupation time on $[0, \infty)$ for skew two-sided Bessel diffusion processes. More precisely, let $\left(X_{t}\right)_{t \geqslant 0}$ be a skew two-sided Bessel process $[2,12,13]$ starting at $X_{0}=0$ with skewness 
parameter $x \in(0,1)$ and parameter $z \in(-1,0)$ related to the dimension of the process $(d=2+2 z)$, and let further $A_{t}$ be the occupation time on $[0, \infty)$ of the process up to time $t$,

$$
A_{t}:=\int_{0}^{t} \mathbb{1}_{[0, \infty)}\left(X_{s}\right) \mathrm{d} s .
$$

Then, the $n$th moment $\mathbb{E}\left(A_{1}^{n}\right)$ of the occupation time up to time 1 is equal to $P_{n}(x, z)$, which is given recursively by (10) and explicitly in (11), as shown in [12, Thms. 3, 4]. Note that, due to self-similarity of the Bessel processes, it follows that $\mathbb{E}\left(A_{t}^{n}\right)=t^{n} P_{n}(x, z)$ for all $t \geqslant 0$.

The special case when $z=-1 / 2$ corresponds to a skew Brownian motion. This is precisely the case considered in the proof of Theorem 1. In fact the proof was attained by first solving the recurrence relation (12) for a skew Brownian motion and later using another path to solve the more general recurrence for skew two-sided Bessel processes. Comparing the results then led to the interesting identity (2). As described in Section 2, this identity is found in literature $[11,14]$, although in other sources it is proved using different approaches.

The moments of $A_{1}$ for a skew Brownian motion with skewness parameter $x \in(0,1)$ is thus given by (13), which alternatively can be expressed using Bessel numbers as

$$
\mathbb{E}\left(A_{1}^{n}\right)=\frac{1}{2^{n-1}(n-1) !} \sum_{k=1}^{n}(-1)^{n-k}(k-1) ! x^{k} b(n, k) .
$$

The sum somewhat resembles a modified version of an inverse Bessel polynomial, since

$$
x \theta_{n-1}(x)=\sum_{k=1}^{n}(-1)^{n-k} x^{k} b(n, k) .
$$

However, the recurrence for $\mathbb{E}\left(A_{1}^{n}\right)$ as given in (12) is naturally very different from the simple recurrence for inverse Bessel polynomials in (5).

As noted in the introduction, Theorem 1 is also a special case of (9). If we let $\sigma=1$ and $\nu=s+1$ this equation becomes

$$
\mathfrak{S}_{\frac{s}{s+1} ; s+1}(n, k)=\sum_{i=k}^{n} \mathfrak{S}_{1 ; s}(n, i) \mathfrak{S}_{0 ; 1}(i, k)=\sum_{i=k}^{n} s^{n-i}\left[\begin{array}{l}
n \\
i
\end{array}\right]\left\{\begin{array}{l}
i \\
k
\end{array}\right\},
$$

and, writing $z=s^{-1}$, we obtain

$$
\sum_{i=k}^{n}\left[\begin{array}{l}
n \\
i
\end{array}\right]\left\{\begin{array}{l}
i \\
k
\end{array}\right\} z^{i}=z^{n} \mathfrak{S}_{\frac{1}{z+1} ; \frac{z+1}{z}}(n, k) .
$$

Thus, for any skew two-sided Bessel process with parameter $z \in(-1,0)$, the moments of the positive occupation time can alternatively be expressed using generalized Stirling 
numbers as

$$
\begin{aligned}
\mathbb{E}\left(A_{1}^{n}\right) & =\sum_{k=1}^{n} \frac{(-1)^{k-1}(k-1) ! x^{k}}{x(n-1) !} \sum_{i=k}^{n}\left[\begin{array}{l}
n \\
i
\end{array}\right]\left\{\begin{array}{l}
i \\
k
\end{array}\right\} z^{i} \\
& =\frac{z^{n-1}}{(n-1) !} \sum_{k=1}^{n}(-1)^{k-1}(k-1) ! x^{k} \mathfrak{S}_{\frac{1}{z+1}, \frac{z+1}{z}}(n, k),
\end{aligned}
$$

which has a structure similar to (22). This illustrates that Bessel numbers occur in the moments of occupation times on $[0, \infty)$ for a skew Brownian motion, while generalized Stirling numbers are, correspondingly, found in the more general case of skew two-sided Bessel processes.

\section{Acknowledgements}

The author wishes to thank Professor James Wan for his valuable comments on the case with negative integer $z$, as well as Professor Christophe Vignat for suggesting the alternative approach using generating functions as presented in Remark 6 .

\section{References}

[1] Abramowitz, M. and Stegun, I. A. (1966). Handbook of mathematical functions, with formulas, graphs, and mathematical tables. Dover Publications, Inc., New York.

[2] Barlow, M., Pitman, J. and Yor, M. (1989). Une extension multidimensionnelle de la loi de l'arc sinus. In: Séminaire de Probabilités, XXIII. Lecture Notes in Math., Vol. 1372, pp. 294-314. Springer, Berlin.

[3] Chu, W. (2010). Elementary proofs for convolution identities of Abel and HagenRothe. Electron. J. Combin. 17, \#N24.

[4] Comtet, L. (1974). Advanced combinatorics D. Reidel Publishing Company, Dordrecht.

[5] Gould, H. W. (1972). Combinatorial identities. Henry W. Gould, Morgantown.

[6] Graham, R. L., Knuth, D. E. and Patashnik, O. (1994). Concrete mathematics, 2nd ed. Addison-Wesley Publishing Company, Reading.

[7] Grosswald, E. (1978). Bessel polynomials. Lecture Notes in Math., Vol. 698. Springer, Berlin.

[8] Knuth, D. E. (1992). Two notes on notation. Amer. Math. Monthly 99, 403-422.

[9] Krall, H. L. and Frink, O. (1949). A new class of orthogonal polynomials: The Bessel polynomials. Trans. Amer. Math. Soc. 65, 100-115.

[10] Mansour, T. and Schork, M. (2016). Commutation relations, normal ordering, and Stirling numbers. CRC Press, Boca Raton.

[11] Mansour, T., Schork, M. and Shattuck, M. (2012). The generalized Stirling and Bell numbers revisited. J. Integer Seq. 15, Article 12.8.3. 
[12] Salminen, P. and Stenlund, D. (2021). On occupation times of one-dimensional diffusions. J. Theor. Probab. 34, 975-1011.

[13] Watanabe, S. (1995). Generalized arc-sine laws for one-dimensional diffusion processes and random walks. In: Cranston, M. C. and Pinsky, M. A. (eds.) Stochastic analysis (Ithaca, NY, 1993). Proc. Sympos. Pure Math. Vol. 57, pp. 157-172. Amer. Math. Soc., Providence.

[14] Yang, S. L. and Qiao, Z. K. (2011). The Bessel numbers and Bessel matrices. J. Math. Res. Exposition 31, 627-636. 